Léonie Chinet

Bernard Plancherel

Monique Bolognini

Mathieu Bernard

Jacques Laget

Giusi Daniele

Olivier Halfon

\section{Substance use and depression. Comparative course in adolescents}

Accepted: 7 November 2005 /

Published online: 10 March 2006
L. Chinet, MA $(\bowtie) \cdot B$. Plancherel

M. Bolognini - M. Bernard · J. Laget

G. Daniele · O. Halfon

SUPEA Research Unit

University Child and Adolescent

Psychiatric Clinic

University of Lausanne

Bugnon $25 \mathrm{~A}$

1005 Lausanne, Switzerland

Tel.: +41-21/314-74-88

Fax: +41-21/314-74-81

E-Mail: lchinet@hospvd.ch
Abstract Objective To examine the relation between depression and substance use in adolescents and the concomitant courses of both disorders. Methods Four individual interviews were administered to 85 adolescent substance users aged 14-19 years (mean 17.1 years, SD 1.4) over a 3.5 year period using the Adolescent Drug Abuse Interview (ADAD) and the Beck Depression Inventory (BDI13). Results No predictive effect was observed on one dimension over the other, but each dimension was predictive of its own course. A decrease in substance-use severity paralleled a decrease in depressive state. Similarly, stable substanceuse rates, either at a low or a high level, tended to be associated with low or high levels of depression, respectively. However, an increase in substance use was not accompanied by an increase in depressive states. Moreover, depression varied greatly between adolescents, and according to gender and age. Conclusions Depressive states and substance use in adolescents can vary considerably overtime, and are closely but rather synchronically related. Since most of the adolescents do not seek help for substance-related problems, substance use should be systematically assessed in adolescents presenting with a depressive state.

Key words adolescent - substance use - depression - longitudinal design - community study

\section{Introduction}

Adolescence is a key period with regard to substance use. The risk of substance-use onset is highest in this age group [18]. The earlier the onset of substance use, the greater the risk of future dependence [17]. Furthermore, substance use in adolescents may lead to impairments that will interfere with normal development, which might, in turn, reinforce substance use $[7,12,17,21]$. Although the association between substance-use disorders and depression has been consistently established, little evidence regarding the underlying mechanisms behind this association is currently available $[9-11,23]$. The present study sets out to explore the concurrent natural course of substance use and depressive state in a community-based sample of regular adolescent substance users.

As in other European countries, substance-use trends in Switzerland show a disturbing increase in teenagers. In 2002, lifetime cannabis use was $49.9 \%$ in boys and $39.1 \%$ in girls aged 15-16 years, which represents a $20 \%$ increase since 1994 . Daily cannabis use is also increasing, with $6.5 \%$ of adolescents reporting daily use in recent years [16]. Moreover, patterns of substance use are changing toward more 
frequent, risky behaviours such as binge drinking, daily cannabis use, and multiple substance use on the same occasion $[15,16,24]$.

Both community-based and clinical studies show the large co-morbidity between substance-use disorders and other psychiatric disorders, especially anxiety and depression, in adult as well as adolescent populations [2, 5, 18-20]. Prevalence rates of depression of between 20 and $30 \%$ are reported in adolescent substance users [2].

Four models have been proposed to explain the relation between substance use and psychiatric disorders [23]. The first model asserts that high rates of co-morbidity are the result of shared underlying factors, such as genetic factors or personality disorders, inducing common vulnerability. The second model postulates that substance use is secondary to psychiatric disorders, and notably that substance use could be a way of alleviating 'feeling bad' (i.e. affect regulation model). In contrast, the third model posits that psychiatric illness is induced by primary substance use. Finally, the fourth model asserts that interacting effects accounting for the increased co-morbidity rates could link both disorders.

The objective of this study was to investigate the relation between depression and substance use in adolescents and the concomitant courses of both disorders. In particular, the study sets out to examine the hypotheses referring to the common factor model, the affect regulation model, the secondary psychiatric disorder model and the bi-directional model.

\section{Methods}

\section{Population}

The sample at baseline included 102 adolescents (66 boys and 36 girls aged $14-19$ years, 17.1 years SD 1.4) recruited between January 1999 and March 2000 in the French-speaking part of Switzerland, an area with approximately one million inhabitants. They all fitted the criterion of regular illicit drug or alcohol use (at least one substance once a week during the last 3 months) at the first interview (time 1). All of them were regular cannabis users. Ninety-four per cent fitted the DSM-IV (Diagnostic and Statistical Manual of Mental Disorders version 4) criteria for cannabis abuse or dependence, of which $54 \%$ received additional substance-abuse diagnoses, mainly for alcohol (38\%). Seventy-five percent were Swiss and 25\% came from another country (mainly European), which corresponds to the percentages observed in the general population. Eighty-nine percent of the subjects were native French speakers. None of the subjects was excluded due to language difficulties or because of psychosis or chronic physical disease, which were exclusion criteria. Regarding family background, $63 \%$ of the subjects had lived most of their life with both parents whereas $16 \%$ had lived mainly with their mother alone. The remaining $21 \%$ had lived either in a mixed family (11\%); with adoptive parents, other relatives, or a foster family $(6 \%)$; or in community homes $(4 \%)$. They were recruited in outpatient units $(26 \%)$, residential units $(27 \%)$, hospitals $(15 \%)$ and leisure places $(32 \%)$.

\section{Procedure}

Adolescents were recruited in a variety of contexts, through collaboration with youth-service professionals, through dissemination and direct distribution of flyers in public and leisure places, or through direct contact. They were informed about the conditions of participation before they joined the study, in particular the fact that they were free to leave the study at any time. They were given a SFr. 50.00 incentive for the time spent on each interview. They all gave written consent and Ethical Committee approval was obtained. Three consecutive interviews, hereafter referred to as time 2, time 3 and time 4, were administered after 9, 18 and 42 months. Drop-out rates were $17 \%$ at time 3 and $19 \%$ at time 4 . Most of the dropouts were due to loss of contact because of moving or telephone number changes. Three adolescents left the study on their own. The time 3 and time 4 samples were comparable to the baseline sample according to age $(F[1,100]=1.728, P=0.192$ and $F[1,100]=$ $0.002, P=0.961$, respectively), gender $\left(\chi^{2}=0.4\right.$, $P 2=0.1, \quad P=0.74$, respectively) and sociodemographic characteristics.

At each time, trained psychologists administered a standard protocol to adolescents individually. Depression was measured using the BDI-13 (Beck Depression Inventory, short version) [3]. The French translation was used [8], which assesses the current depressive state. This instrument is composed of 13 questions with four statements related to depression symptomatology rated on Likert-type scales from 0 to 3 with the total score ranging from 0 to 39 . Total scores ranging from 0 to 4 correspond to "not depressed", those from 5 to 7 to "lighty depressed", those from 8 to 15 to "fairly depressed" and those of 16 and over to "severely depressed". In this study, the total score was used in order to study depressive state changes over time compared to substance-use evolution. The French version of the ADAD (Adolescent Drug Abuse Diagnosis) [4] was used to evaluate illegal drug and alcohol use and associated life problem areas. For each domain, interviewers attribute severity-rating scores ranging from 0 (no real problem) to 
9 (extreme problem, treatment absolutely necessary) on the basis of the adolescents' reports and self-perception of their situation. A severity rating score of 4 and over indicates that the problems in this area are important and necessitate treatment. Finally the MINI (Mini International Neuropsychiatric Interview) [25] was used, which provides substance-abuse diagnoses based on DSM-IV criteria.

\section{Data transformation}

Three transformations were performed before analyzing the data. First, BDI mean scores showed a high variability with irregular variations across gender and substance-use evolution subgroups (SUES; see Table 1 in the Results). Therefore BDI individual scores were transformed into natural logarithm scores (hereafter mentioned as BDI ln mean scores), and scores equal to zero were replaced manually. The transformation to BDI ln mean scores made the variation smaller and more constant across levels of data series. BDI ln scores were thus used in the subsequent statistical analyses. Second, age was dichotomised into adolescents aged under 17 years and those aged 17 and over to introduce this variable into the general linear model (GLM) analyses as a between-subjects factor. The younger and older age groups contained 46 and 39 adolescents, respectively. Finally, the grouping analysis of Wills et al. [27] was used to explore the courses of both depression and substance use simultaneously over time according to gender and age. Therefore, clustering analysis using individual substance-use severity scores at times 1,2 and 3 was performed to determine empirical patterns of substance-use over time. The depression rate of drop-outs at baseline was significantly higher compared to those who completed the time 4 interview. Moreover, subjects' age at time 4 ranging from 18- to 23-years old made them more comparable to young adults than adolescents, and the time frame between time 4 and time 3 differed from the others. For these reasons, the clustering analysis was conducted on the 85 adolescents who completed the first three interviews. The analysis produced four SUES: one group of 15 adolescents, referred to as increasers (IC), whose substance use increased over time; one group of 22 adolescents, referred to as decreasers (DC), whose substance use decreased over time; and two groups with either low or high stable substance-use severity, one group of 18 low persisters (LP) and one composed of 30 high persisters (HP). Cluster membership was cross-validated by making sure that increasers' and decreasers' substance-use score changes were of 2 points or more. These substance-use evolution patterns were confirmed by substance-use trends in each SUES at time 4, with IC and HP presenting significantly higher substance-use severity scores than $\mathrm{DC}$ and $\operatorname{LP}(F[3,74]=6.98, P=0.000)$.

Table 1 Depression and substance-use mean scores according to substance-use evolution subgroups (SUES), gender and age

\begin{tabular}{|c|c|c|c|c|c|c|c|c|c|}
\hline & & \multicolumn{4}{|c|}{ BDI mean scores* } & \multicolumn{4}{|c|}{ ADAD drug severity mean scores } \\
\hline & & time 1 & time 2 & time 3 & Mean & time 1 & time 2 & time 3 & Mean \\
\hline \multicolumn{10}{|l|}{ SUES } \\
\hline \multirow{2}{*}{ Increasers $(n=15)$} & Mean & 7.53 & 9.10 & 8.47 & 8.37 & 4.47 & 5.73 & 6.93 & 5.71 \\
\hline & SD & 5.04 & 6.82 & 6.10 & 5.11 & 1.51 & 1.03 & 0.96 & 0.93 \\
\hline \multirow[t]{2}{*}{ Decreasers $(n=22)$} & Mean & 9.05 & 6.11 & 3.86 & 6.28 & 6.23 & 4.50 & 2.55 & 4.42 \\
\hline & SD & 5.18 & 4.85 & 4.23 & 3.89 & 1.60 & 2.54 & 1.34 & 1.49 \\
\hline \multirow[t]{2}{*}{ Low persisters $(n=18)$} & Mean & 6.06 & 5.00 & 4.78 & 5.28 & 3.67 & 3.39 & 3.33 & 3.46 \\
\hline & SD & 6.99 & 7.88 & 6.98 & 6.59 & 1.61 & 1.75 & 1.68 & 1.60 \\
\hline \multirow{2}{*}{ High persisters $(n=30)$} & Mean & 10.30 & 8.31 & 8.62 & 9.12 & 6.87 & 5.86 & 6.77 & 6.47 \\
\hline & SD & 7.96 & 6.88 & 7.91 & 6.52 & 1.07 & 1.43 & 1.07 & 1.15 \\
\hline \multicolumn{10}{|l|}{ Gender } \\
\hline \multirow[t]{2}{*}{ Girls $(n=30)$} & Mean & 10.80 & 8.97 & 7.29 & 9.01 & 5.90 & 4.76 & 4.73 & 5.18 \\
\hline & SD & 8.13 & 9.19 & 7.54 & 7.50 & 2.34 & 2.32 & 2.57 & 2.14 \\
\hline \multirow[t]{2}{*}{ Boys $(n=55)$} & Mean & 7.38 & 6.24 & 6.15 & 6.56 & 5.44 & 5.05 & 5.11 & 5.16 \\
\hline & SD & 5.56 & 4.67 & 6.48 & 4.54 & 1.63 & 1.87 & 2.23 & 1.53 \\
\hline \multicolumn{10}{|l|}{ Age } \\
\hline \multirow[t]{2}{*}{ Youngers $(n=46)$} & Mean & 7.15 & 6.15 & 5.47 & 6.22 & 5.28 & 5.04 & 4.83 & 5.05 \\
\hline & SD & 5.65 & 5.39 & 5.11 & 4.26 & 1.60 & 1.76 & 2.17 & 1.35 \\
\hline \multirow{2}{*}{ Olders $(n=39)$} & Mean & 10.28 & 8.42 & 7.82 & 8.87 & 5.97 & 4.84 & 5.15 & 5.31 \\
\hline & SD & 7.56 & 7.86 & 8.35 & 7.07 & 2.18 & 2.33 & 2.56 & 2.15 \\
\hline \multirow[t]{2}{*}{ Total $(n=85)$} & Mean & 8.59 & 7.20 & 6.55 & 7.44 & 5.60 & 4.95 & 4.98 & 5.17 \\
\hline & SD & 6.74 & 6.70 & 6.85 & 5.84 & 1.91 & 2.03 & 2.35 & 1.76 \\
\hline
\end{tabular}

*BDI mean scores ranging from 0 to 4 correspond to "not depressed", from 5 to 8 to "lightly depressed", from 9 to 15 to "fairly depressed" and 16 and over to "severely depressed" 


\section{Statistical analyses}

Preliminary analyses are presented that explore depression, substance use and individual factors such as gender, age and substance-use duration. Structural equation modeling, using Amos software [1], was then performed to investigate predictive relations between depression and substance use. Finally, GLM with repeated measures were performed using BDI ln mean scores at each time measure as the dependent variable and SUES, age groups and gender as factors. These analyses allowed the appraisal of the joint evolution of both dimensions.

\section{Results}

Table 1 presents BDI and ADAD substance-use severity rating mean scores according to SUES, gender and age at time 1,2 and 3.

Drug severity rating total mean scores were relatively high, indicating a need for some treatment, except in the LP group. Older adolescents tended to be rated slightly higher than younger adolescents for drug severity, but only at baseline $(F[1,83]=2.83$, $P=0.096)$. There was no difference in drug severity according to gender. Concerning depression, BDI total mean scores showed an overall moderate depressive state (between lightly and fairly depressed according to BDI scores grouping). Depressive rates differed at baseline according to gender, girls being significantly more depressed than boys $(F[1,83]=$ 5.25, $P=0.024)$. However, this discrepancy diminished over time and was not found in BDI total mean scores. With respect to age groups, older adolescents tended to be globally more depressed than the younger adolescents $(F[1,83]=3.54$, $P=0.063)$, which was especially the result of depression rates at baseline (2.00 SD 0.94 and 1.64 SD 0.89 , respectively). SUES were then compared according to potential influential factors. Substanceuse duration varied according to SUES ( $F$ $[3,81]=2.58, P=0.059)$. HP had been using substances since a longer period of time compared to LP (2.8 years, SD 1.4 and 2.1 years SD 0.93 , respectively). However no difference appeared among SUES according to age.

Structural equation modeling was then performed to investigate predictive relations between depression and substance use. Auto-regressive cross-lagged panel models were performed with BDI ln and substanceuse severity rating mean scores at each time point. According to the alternative hypotheses presented in the introduction, two a priori models were tested, one with cross-lagged inter-dimensional paths linking prior substance use with successive depres-

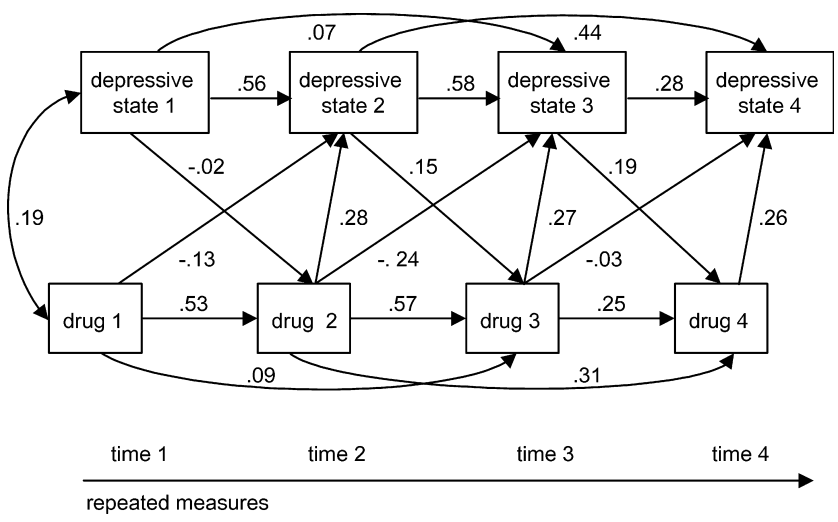

Fig. 1 Effects of prior depression (BDI In mean scores) and substance-use severity (ADAD mean scores) on successive measures. One-tailed arrows are path-coefficients and the two-tailed is a correlation

sion-corresponding to the 'secondary mood disorder model' - and one with paths between prior depression and substance use-according to the 'affect regulation model'. Both models failed to meet the criteria for adequate fit. However, when both models were combined and 2-lag intra-dimensional paths were added, all criteria indicated a good model fit (c2 = 11.97, df $8, P=0.153$, RMSEA 0.07).

Fig. 1 presents this model comprising 1- and 2-lag intra-dimensional paths and 1-lag inter-dimensional paths, showing that both depressive state and substance use were significantly predictive of each future respective course. Globally direct effects within each dimension predominated compared to cross-lagged inter-dimensional path coefficients. Inter-dimensional path coefficients were, overall, relatively low, except for one negative coefficient between substance use at time 2 and depression at time 3 . Simple linear regression showed, however, that the residual part of substance use 2 in predicting BDI $\ln 3$ was near zero once BDI ln 2 was controlled for. Finally, coefficients were moderately high between both dimensions within the same time measure.

GLM with repeated measures were performed using time 1 to time 3 BDI ln mean scores as dependent variables and SUES, age groups and gender as factors. These analyses allowed appraisal of the joint evolution of both dimensions.

A time effect appeared in the GLM analysis $(F[2,134]=4.64, P=0.011)$, which indicated that the depressive state tended to decrease over time (see Fig. 2). There was a tendency towards differentiated evolution patterns for depression according to substance-use subgroups $(F[6,134]=1.94, P=0.079)$. The depressive state decreased dramatically in the DC group whereas it remained stable at a high level in the HP and IC groups, and at a low level in the LP group. Two significant effects appeared in triple interactions 


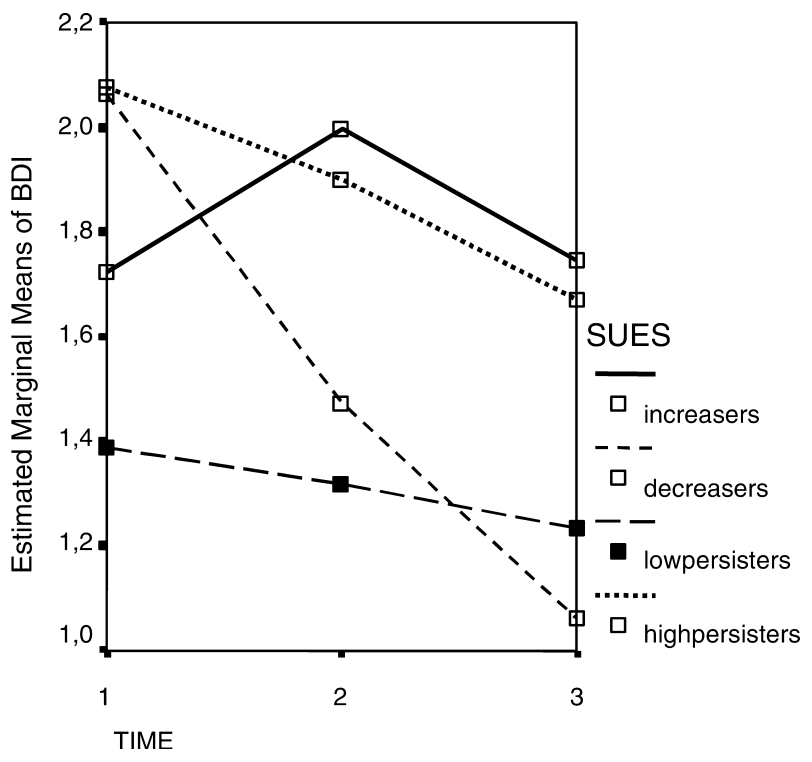

Fig. $2 \mathrm{BDI}$ mean scores at time 1, 2 and 3 by substance-use evolution subgroups (SUES)

with time, SUES and gender $(F[6,134]=2.70$, $P=0.017)$, and with time, gender and age $(F[1,67]=5.61, P=0.021)$. Older girls presented with higher depressive states at baseline, which then tended to show a greater decrease over time compared to the other adolescents. Three between-subjects effects occurred, two tendencies with SUES $(F[3,67]=2.49$, $P=0.068)$ and gender $(F[1,67]=3.60, P=0.062)$, and one significant effect with age $(F[1,67]=5.90$, $P=0.018$ ). Girls and older subjects appeared to be more depressed than boys and younger subjects, respectively. Furthermore, there was an interaction of gender with age $(F[1,67]=4.19, P=0.045)$, older girls being more depressed than all other adolescents.

Regarding the difference in depression total mean scores over time according to SUES, post-hoc tests performed in the one-way analysis comparing BDI total mean scores by subgroups showed a significant difference between the LP and HP groups, the former being globally less depressed than the latter (see Table 1). At time 4, the depressive state in the LP group remained significantly lower than that in the HP group, and was also significantly lower than those in the IC and DC groups (1.15 SD 0.99, 6.62 SD 5.13, 7.30 SD 5.12 and 5.70 SD 4.39, respectively, $F[3,65]=5.29$, $P=0.003)$.

\section{Discussion}

The different patterns evidenced overtime show that depression and substance use may vary considerably during adolescence. Regarding changes over time in depression and substance use, there was no predictive relation of one dimension over the other. Instead, prior depressive states and substance-use severity were each predictive of successive measures in the respective dimensions. Moreover, when analysing the substance-use evolution subgroups separately, depression varied over time according to substanceuse evolution patterns, gender and age. In every case, a decrease in substance-use severity paralleled a decrease in depressive state. In the same way, stable substance-use subgroups, either with a low or a high level of substance-use severity, tended to present stable low or high levels of depression, respectively. An increase in substance use was not accompanied by an increased depressive state, which can be explained by relatively high depressive states at baseline. The lack of prediction of one dimension over the other argues against both secondary psychiatric disorder-and affect regulation explanation models [23]. Whereas the common factor model cannot be definitely proved false by our results, they give more support to the interacting effects model.

Globally depressive state differed according to gender and age, girls-especially older girls-being significantly more depressed than boys. However, depression rates were rather heterogeneous and varied over time, particularly in those groups where depression was highest at baseline. These results paralleled those found in the literature $[6,10,11,28]$.

Substance misuse was associated with relatively high depressive states. Moreover girls, and especially older ones, presented more severe, but highly heterogeneous, depressive states.

There were no predictive relations for one dimension over the other. Prior depression rates predicted successive depression rates, which was also the case for substance-use severity. Moreover, depressive states and substance-use severity were moderately associated at each time point. The importance of these relations, on the one hand within each dimension across time and on the other hand in cross-dimensional paths at each time point, could induce an artefact accounting for the negative coefficients found in two of the cross-lagged paths. This explanation is supported by the lack of a 'singular' predictive residual part of substance-use once prior depression is controlled for.

Depression course was then analysed according to substance-use evolution patterns, gender and age. A general observation was that a decrease in substanceuse severity was accompanied by a decrease in depressive symptoms. Moreover, adolescents whose substance-use remained stable either at a low (LP) or a high (HP) level tended to present similarly low and high depressive rates. Depressive states and substance 
use in adolescents appear to be closely, but rather synchronically, related. This could be explained by the fact that each problem, and its associated impairments, might contribute to reinforce the other in a reciprocal way. These observations are consistent with those of Kandel et al. [19]; they support the usefulness of detecting substance misuse and depressive states at an early stage to provide adolescents with an integrated-treatment plan that will target both disorders and help prevent mutual relapse $[13,14]$.

Some limitations should be mentioned regarding the results discussed here. First, depression rates showed an important variability, with some adolescents having extremely high scores, especially at baseline where one-third of the sample was recruited from within health or social services. This could explain the general tendency towards a decrease in depression rates (regression toward the mean), and the fact that an increase in substance use was not accompanied by an increase in depressive state. Second, the respective courses of depression and substance use were evaluated when substance use was already established. Thus depressive traits might be present prior to substance-use onset and vice versa. Third, the intervals between each time measure were large. Consequently, it might be the case that a causal relation exists between the courses of both disorders, but within a shorter timescale.

Nevertheless these results show that some of the depressive symptoms could be directly associated with substance-use severity, especially in boys [28]. Regarding the treatment of depressive states in adolescents, this observation stresses the importance of carefully assessing substance use and taking it into account in the treatment planning. Moreover, current pharmacological treatments for adolescent depression were not found to be particularly effective, notably in substance-user adolescents [22], and a 6-8 week period was required before any effect was seen [26]. Further research is needed to follow both disorder courses with shorter time intervals between each measure in order to explore in-depth an eventual causal relation.

Acknowledgments This study was supported by a research grant from the Swiss Federal Office of Public Health, ref. 316.98.8108.

\section{References}

1. Arbuckle JL (1989) Amos: Analysis of moment structures. Am Stat 43:66-67

2. Armstrong TD, Costello EJ (2002) Community studies on adolescent substance use, abuse, or dependence and psychiatric comorbidity. J Consult Clin Psychol 70:1224-1239 [Review]

3. Beck AT, Beamesderfer A (1974) Assessment of depression: the depression inventory. Mod Probl Pharmacopsychiatry 7:151-169

4. Bolognini M, Plancherel B, Laget J, Chinet L, Rossier V, Cascone P, Stéphan P, Halfon O (2001) Evaluation of the Adolescent Drug Abuse Diagnosis instrument in a Swiss sample of drug abusers. Addiction 96:1477-1484

5. Brook DW, Brook JS, Zhang CS, Cohen P, Whiteman M (2002) Drug use and the risk of major depressive disorder, alcohol dependence, and substance use disorders. Arch Gen Psychiatry 59: 1039-1044

6. Brook JS, Cohen P, Brook DW (1998) Longitudinal study of co-occurring psychiatric disorders and substance use. J Am Acad Child Adolesc Psychiatry $37: 322-330$
7. Chander G, McCaul ME (2003) Cooccurring psychiatric disorders in women with addictions. Obstet Gynecol Clin North Am 30:469-481

8. Collet L, Cottraux J (1986) [The shortened Beck depression inventory, (13 items). Study of the concurrent validity with the Hamilton scale and Widlocher's retardation scale]. Encephale 12:77-79 [French]

9. Costello EJ, Erkanli A, Federman E, Angold A (1999) Development of psychiatric comorbidity with substance abuse in adolescents: effects of timing and sex. J Clin Child Psychol 28:298311

10. Degenhardt LH (2003) Exploring the association between cannabis use and depression. Addiction 98:1493-1504

11. Enns MW, Swenson JR, McIntyre RS, Swinson RP, Kennedy SH (2001) Clinical guidelines for the treatment of depressive disorders VII. Comorbidity. Can J Psychiatry-Revue Canadienne de Psychiatrie 46:77S-90S
12. Friedman AS, Bransfield S, Kreisher C (1994) Early teenage substance use as a predictor of educational-vocational failure. Am J Addict 3:325-336

13. Giaconia RM, Reinherz HZ, Paradis AD, Hauf AM, Stashwick CK (2001) Major depression and drug disorders in adolescence: general and specific impairments in early adulthood. J Am Acad Child Adolesc Psychiatry 40:1426-1433

14. Grella CE, Hser YI, Joshi V, RoundsBryant J (2001) Drug treatment outcomes for adolescents with comorbid mental and substance use disorders. J Nerv Ment Dis 189:384-392

15. ISPA (Institut suisse de prévention de l'alcoolisme et autres toxicomanies) (2001) Alcool, tabac et cannabis dans la réalité quotidienne des 12-15 ans. Sélection de résultats d'une enquête menée sous l'égide de l'OMS avec le soutien financier de l'OFSP. ISPA, Lausanne

16. ISPA (Institut suisse de prévention de l'alcoolisme et autres toxicomanies) (2003) Chiffres et données 2003. ISPA, Lausanne 
17. Kandel DB, Davies M, Karus D, Yamaguchi K (1986) The consequences in young adulthood of adolescent drug involvement. An overview. Arch Gen Psychiatry 43:746-754

18. Kandel DB, Huang FY, Davies M (2001) Comorbidity between patterns of substance use dependence and psychiatric syndromes. Drug Alcohol Depend 64:233-241

19. Kandel DB, Johnson JG, Bird HR, Weissman MM, Goodman SH, Lahey BB (1999) Psychiatric comorbidity among adolescents with substance use disorders: Findings from the MECA study. J Am Acad Child Adolesc Psychiatry 38:693-699

20. Kessler RC, Nelson CB, McGonagle KA, Edlund MJ, Frank RG, Leaf PJ (1996) The epidemiology of co-occurring addictive and mental disorders: Implications for prevention and service utilization. Am J Orthopsychiatry 66:17-31
21. Lynskey MT, Coffey C, Degenhardt L, Carlin JB, Patton G (2003) A longitudinal study of the effects of adolescent cannabis use on high school completion. Addiction 98:685-692

22. Milin R, Walker S, Chow J (2003) Major depressive disorder in adolescence: a brief review of the recent treatment literature. Can J Psychiatry 48:600-606 [Review]

23. Mueser KT, Drake RE, Wallach MA (1998) Dual diagnosis: a review of etiological theories. Addict Behav 23:717734 [Review]

24. Narring F, Tschumper A, Inderwildi Bonivento L, Jeannin A, Addor V, Bütikofer A (2003) Santé et styles de vie des adolescents âgés de 16 à 20 ans en Suisse, (2002). SMASH 2002: Swiss Multicenter Adolescent Study on Health 2002. Institut Universitaire de Médecine Sociale et Préventive Lausanne
25. Sheehan DV, Lecrubier Y, Sheehan KH, Amorim P, Janavs J, Weiller E (1998) The Mini-International Neuropsychiatric Interview (M.I.N.I.): the development and validation of a structured diagnostic psychiatric interview for DSM-IV and ICD-10. J Clin Psychiatry 20(59):22-33 [Review]

26. Thorpe L, Whitney DK, Kutcher SP, Kennedy SH (2001) Clinical guidelines for the treatment of depressive disorders VI. Special populations. Can J Psychiatry 46:63S-76S

27. Wills TA, Vaccaro D, McNamara G, Hirky AE (1996) Escalated substance use: a longitudinal grouping analysis from early to middle adolescence. J Abnorm Psychol 105:166-180

28. Zilberman ML, Tavares H, Blume SB, el Guebaly N (2003) Substance use disorders: Sex differences and psychiatric comorbidities. Can J Psychiatry 48:5-13 